\title{
Влияние эффекта перколяции на температурные зависимости вольт-фарадных характеристик гетероструктур на основе композитных слоев наночастиц кремния и золота
}

\author{
() М.М. Соболев, Д.А. Явсин, С.А. Гуревич \\ Физико-технический институт им. А.Ф. Иоффе Российской академии наук, \\ 194021 Санкт-Петербург, Россия \\ E-mail:m.sobolev@mail.ioffe.ru
}

Поступила в Редакцию 31 мая 2019 г.

В окончательной редакции 10 июня 2019 г.

Принята к публикации 10 июня 2019 г.

Исследованы температурные зависимости вольт-фарадных характеристик и спектры глубоких уровней гетероструктуры $\mathrm{Au}-n-\mathrm{Si}: \mathrm{Au}-\mathrm{Si}-p-\mathrm{Si}$ на основе композитного слоя наночастиц $\mathrm{Au}$ и $\mathrm{Si}$. При температуре $300 \mathrm{~K}$ структура проявляет свойства транзистора, включенного по схеме с общим эмиттером при отключенной базе и с эмиттерным барьером Шоттки между точечным контактом Аи и слоем $n-(\mathrm{Si}: \mathrm{Au})$. В этом слое наночастицы образуют конечные кластеры, где проводимость будет прыжковой, при этом в области точечного контакта Аи наблюдается аккумуляция заряда. При температуре измерения ниже $180 \mathrm{~K}$ в результате эффекта перколяции система из фазы конечного кластера переходит в фазу бесконечного кластера, проявляющего металлические свойства в латеральной плоскости гетероструктуры, которая превращается в $p-n$-диод.

Ключевые слова: $C-V$-характеристика, DLTS, композитные наночастицы, золото, кремний.

DOI: $10.21883 /$ FTP.2019.10.48303.9180

\section{1. Введение}

Гетероструктуры с нанокомпозитными слоями близко расположенных гранул аморфных наночастиц кремния и металла, в последнее время рассматриваются в качестве основного элемента при создании новых фотодетекторов с внутренним усилением сигнала, демонстрирующих большие значения спектральной чувствительности, и для детектирования слабых оптических сигналов [1-4]. Перспективность применения таких структур определяется их необычными оптическими и электрическими свойствами, которые зависят от технологии получения структур, выбора композитных слоев и соотношения их состава [2-4]. В работах [2,4] были исследованы композитные слои, состоящие из наночастиц аморфного кремния (nano-Si) и наночастиц золота, полученные лазерным электродиспергированием (ЛЭД). Были проведены исследования температурных зависимостей вольт-амперных характеристик и фоточувствительности структур из композитных слоев наночастиц кремния и золота с различными соотношениями на монокристаллическом кремнии $p$-типа проводимости. Показано, что исследуемые структуры обладают огромной спектральной чувствительностью (до $20 \mathrm{~A} / \mathrm{BT}$ ) при комнатной температуре, которая, по мнению авторов статьи $[2,4]$, вызвана эффектом усиления фотопроводимости в композитном слое с большим количеством золота. Проведенные с помощью атомно-силовой микроскопии (AFM) структурные исследования продемонстрирова-

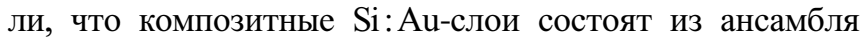

плотноупакованных наночастиц [2]. Исследования транспорта электронов в монодисперсных гранулированных структурах $(\mathrm{Cu}$ и $\mathrm{Ni})$, нанесенные методом лазерной электродисперсии, позволили обнаружить, что проводимость в пленках обусловлена туннельным переносом электронов вблизи порога перколяции [5]. Показано, что для объяснения экспериментальных результатов необходимо использовать модель, в которой рассматривается прыжковая проводимость с переменной длиной прыжка через промежуточные частицы. Аналогичный транспорт носителей наблюдался и в нанослоях кремния (nano-Si). Ранее нами уже проводились исследования методами емкостной спектроскопии $p-n$-гетероструктур со слоями плотноупакованных аморфных наночастиц $\mathrm{Si}$, которые позволили выявить пространственное и квантовое ограничение $\mathrm{Si}$ наночастиц [6]. В случае гетероструктур на основе композитных $\mathrm{Si}: \mathrm{Au}-с л о е в$, состоящих из ансамбля плотноупакованных наночастиц, недостаточно изучена роль этих слоев при формировании $p-n$-перехода и точечного контакта $\mathrm{Cr} / \mathrm{Au}$ к слою $\mathrm{Si}: \mathrm{Au}$. Кроме того, представляется интересным также выявить проявление эффекта перколяции

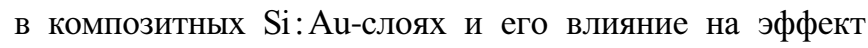
фотоусиления.

Цель данной работы - выявление эффекта перколяции при измерении температурных зависимостей вольтфарадных $(C-V)$ характеристик $p-n$-гетероструктур со слоями плотноупакованных аморфных композитных наночастиц $\mathrm{Si}$ и $\mathrm{Au}$ при высоком содержании золота и влияние эффекта перколяции на фотоусиление исследуемой структуры. 


\section{2. Методики изготовления образцов и измерений}

В настоящей работе методами емкостной спектроскопии исследуется $p-n$-гетероструктура со слоем плотноупакованных аморфных композитных наночастиц $\mathrm{Si}$ и $\mathrm{Au} \mathrm{c}$ соотношениями объемных долей наночастиц кремния $33 \%$ и золота $67 \%$. Слои nano-Si: Аu толщиной $\approx 100$ нм были сформированы на подложке из монокристаллического $\mathrm{Si} p$-типа с предварительно нанесенным на него подслоем nano-Si. Слои наносились с помощью метода модифицированного ЛЭД. Удельное сопротивление подложки было 1-4 Ом.см. Омический контакт к подложке изготавливался посредством термического напыления $\mathrm{Al}$. Точечный контакт к $\mathrm{Si}: \mathrm{Au}$-слою наносился методом магнетронного напыления $\mathrm{Cr} / \mathrm{Au}$ толщиной 20 нм и диаметром 0.5 мм. Исследования температурных зависимостей $C-V$-характеристик и глубоких ловушек в гетероструктурах $\mathrm{Au}-(n-\mathrm{Si}: \mathrm{Au})-\mathrm{Si}-(p-\mathrm{Si})$ проводились методами нестационарной спектроскопии глубоких уровней (DLTS) с помощью спектрометра DL4600 как в темноте, так и при освещении образца белым светом.

\section{3. Результаты экспериментов и их обсуждение}

На рис. $1, a-c$ представлены $C-V$-характеристики гетероструктуры с соотношением состава нанокомпозитных слоев $\mathrm{Si}: \mathrm{Au} \approx 1: 2$ (33\%:67\%), измеренные при приложенном обратном и прямом напряжениях смещения, в темноте и со светом при различных температуpax. При прямом смещении на Аu-контакт подавался отрицательный потенциал относительно $p$-Si-подложки. Емкость диода является функцией приложенного напряжения смещения $U_{b}$. При температуре измерения $300 \mathrm{~K}$ и прямом напряжении смещения $U_{b}>0.5 \mathrm{~B}$ емкость росла. При обратном смещении на Аu-контакт подавался положительный потенциал относительно $p$-Siподложки. В этом случае измерялась барьерная емкость, связанная с изменением потенциального барьера $n-p$-перехода, и при росте напряжения обратного смещения $U_{b}$ до $\approx-0.20 \mathrm{~B}$ емкость незначительно падала. Далее на $C-V$-характеристике наблюдался небольшой участок, где емкость, равная $\approx 33$ пФ, не менялась (рис. $1, a-c)$. С ростом обратного смещения в диапазоне $U_{b} \approx(-0.40--3.0)$ В происходило образование широкого пика, в максимуме которого емкость при $U_{b} \approx-1.77$ В достигала величины $\approx 38$ пФ (рис. $\left.1, a, b\right)$. Слева и справа от этого пика емкость с ростом напряжения смещения показывала медленный рост и падение соответственно. Затем с ростом $U_{b}>3.60 \mathrm{~B}$ емкость увеличилась до 180 п $\Phi$ при $U_{b} \approx-6.70$ В (рис. $1, a, b$ ). Таким образом, $C-V$-характеристика при небольших прямых и обратных напряжениях смещения в диапазоне изменения $U_{b}=(0.6--3.60)$ В проявляла свойства
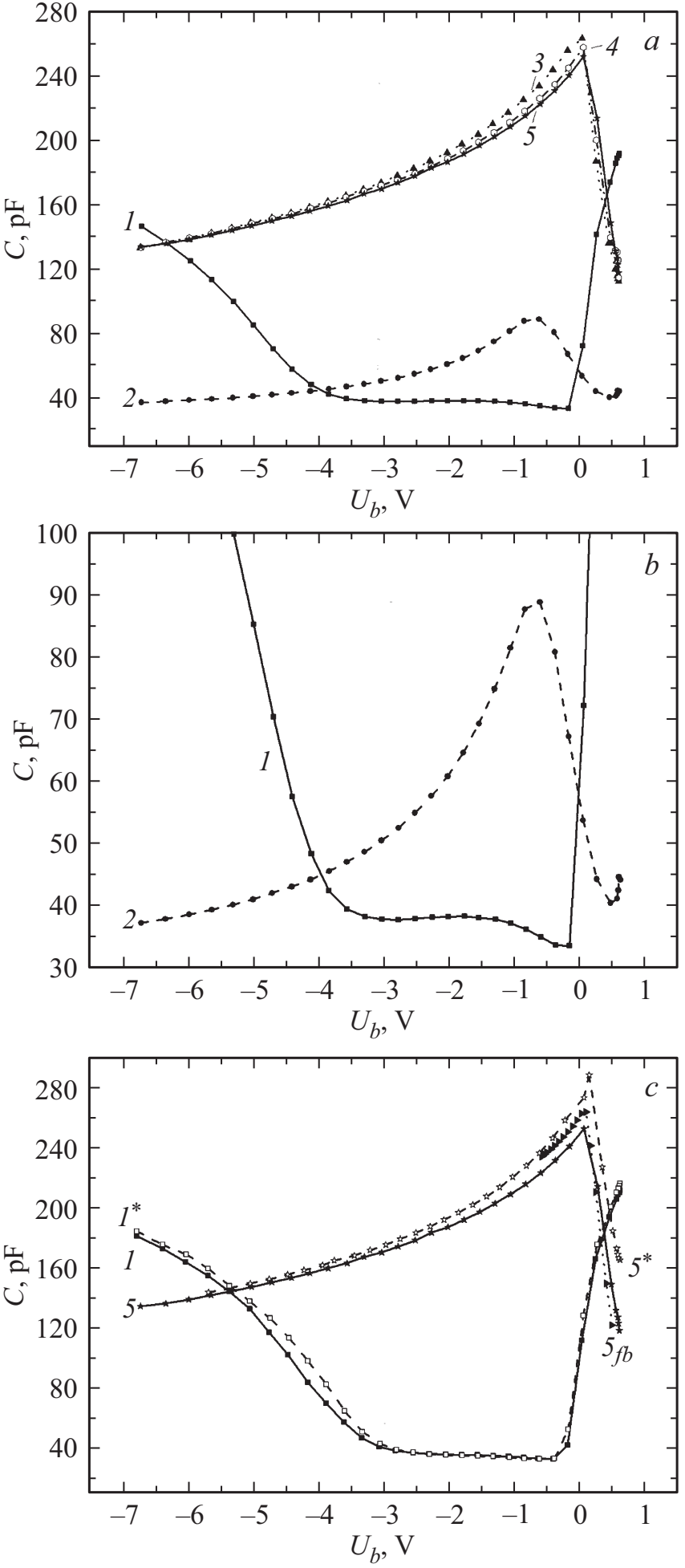

Рис. 1. $C-V$-характеристики $\mathrm{Au}-(n-\mathrm{Si}: \mathrm{Au})-\mathrm{Si}-(p-\mathrm{Si})-$ гетероструктуры, полученной лазерным электродиспергированием: $a, b$ и $c-$ измеренные при различных температурах $T, \mathrm{~K}: 1,1^{*}-300,2-280,3-180,4-130,5,5^{*}-$ 87 ; в темноте $-1-5$, при освещении белым светом $-1^{*}$ и $5^{*}$; при сканировании напряжения смещения из прямого в обратное $-1,1^{*}, 2-5,5^{*}$ и при сканировании из обратного в прямое $-5_{f b}$. 
$p-n$-перехода. При $U_{b}=(-0.20--0.40) \mathrm{B}$, где емкость слабо изменялась, $n$-слой демонстрировал область пространственной локализации носителей (ПЛН), характерную для нанослоев. Подобную область ПЛН мы наблюдали при исследовании $C-V$-характеристик гетероструктуры $\mathrm{Au}-\mathrm{nano}-\mathrm{Si}-p-\mathrm{Si}$ [7]. Если в структуре расположен слой ПЛН, то при смыкании слоя объемного заряда (СО3) с однородным легированием и слоя ПЛН дальнейшему проникновению внешнего электрического поля в полупроводник будет препятствовать экранирование его носителями в слое ПЛН. Поэтому до напряжения, при котором все носители уходят из слоя ПЛН, емкость слабо меняется [6]. Ширина ступеньки $C-V$-характеристики зависит от функции заполнения состояний в слое ПЛН и от положения уровня Ферми в структуре [7]. При подсветке белым светом положение уровня Ферми может изменяться из-за перезарядки поверхностных глубоких уровней (ГУ). Появление максимума на кривой $C-V$-характеристики (при $\left.U_{b} \approx-1.77 \mathrm{~V}\right)$ является свидетельством того, что в слое $\mathrm{Au}-$ nano-Si в области ПЛН имеются глубокие ловушки, в качестве которых могут выступать наночастицы $\mathrm{Si}$ и $\mathrm{Au}$, через которые идет конкурентная тепловая эмиссия и захват электронов на ГУ ловушек, где становится возможным рекомбинация их с дырками [8]. Захваченные на ловушки электроны приводят к увеличению измеряемой емкости. Ток, обусловленный генерацией и рекомбинацией носителей в области обеднения, приводит к увеличению тока утечки и вносит существенный вклад в величину измеряемой емкости в этой области. Темп эмиссия и захвата является функцией величины электрического поля: при малых значениях поля преобладает процесс захвата, с ростом электрического поля доля носителей, эмитирующих с ловушек наночастиц $\mathrm{Si}$ и $\mathrm{Au}$ и уносящих полем, увеличивается, что и отражается в профиле $C-V$-характеристики. Вольт-амперные $(V-A)$ характеристики для этой структуры также свидетельствовали о протекании больших обратных токов [5]. Далее в профиле $C-V$-характеристики с ростом напряжения обратного смещения за областью ПЛН наблюдался участок со значительным градиентом роста емкости, который может быть обусловлен увеличением протекающих через структуру токов утечки, генерацией, рекомбинацией и эффектом накопления носителей вблизи потенциального барьера (рис. 1). Таким барьером может быть барьер Шоттки, образованный Аu-контактом и плотноупакованными композитными слоями наночастиц $\mathrm{Si}$ и $\mathrm{Au}$. Вся структура в таком случае может представлять транзистор с общим эмиттером и отключенной базой, состоящий из $\mathrm{Si}$-подложки $p$-типа, $\mathrm{Si}: \mathrm{Au}$-слоя $n$-типа и барьера Шоттки. Когда коллекторный переход $n-\mathrm{Si}: \mathrm{Au}-p$-Si включен в обратном направлении, то все приложенное напряжение падает на нем, в цепи течет обратный ток коллекторного перехода, включающий в себя ток дырок из базы $(n-\mathrm{Si}: \mathrm{Au})$ в коллектор $(p-\mathrm{Si})$ и ток электронов из коллектора в базу, который приводит к образованию отрицательного заряда в базе [9]. Отрицательный заряд в базе увеличивается и за счет токов генерации и рекомбинации. Вследствие этого потенциальный барьер эмиттера понижается и для его компенсации в базу из эмиттера входят дырки, происходит их аккумуляция вблизи потенциального барьера, которые и дают градиент роста емкости с увеличением напряжения обратного смещения коллекторного перехода. Эта картина $C-V$-характеристики наблюдалась при комнатной температуре $(300 \mathrm{~K})$. При уменьшении температуры измерения $C-V$-характеристики она существенно изменялась. При температуре измерения $280 \mathrm{~K}$ максимум профиля $C-V$-характеристики увеличивался в $\sim 2$ раза, достигая величины $\approx 90$ пФ, и смещался в сторону меньшего значения $U_{b}=-0.64 \mathrm{~B}$ (рис. $\left.1, a, b\right)$. При этом исчезла область накопления носителей вблизи потенциального барьера с характерным ростом емкости при увеличении обратного напряжения смещения. Вместо этого наблюдалось падение емкости с ростом $U_{b}$, что характерно для диффузионной емкости. Дальнейшее понижение температуры измерения привело к еще большему росту емкости в максимуме профиля $C-V$-характеристики, который достигал величины $\approx 263$ пФ, и наблюдался уже при $U_{b}=0.065 \mathrm{~B}$. При дальнейшем увеличении прямого напряжения смещения емкость резко падает, $C-V$-характеристика демонстрирует вид, типичный для $p-n$-перехода с глубокими уровнями. Образование пика в таком случае связывается с существованием электрически активных глубоких ловушек в области гетерограницы $n$-Si: $\mathrm{Au}-p-\mathrm{Si}$ [10]. Имеется и другое объяснение образования пика $C-V$-характеристики при прямом смещении. Оно связывается с протеканием тока, ограниченного пространственным зарядом (SCL), состоящего из суммы дрейфового и диффузионного токов [12]. При прямом смещении с увеличением диффузии инжектированных носителей емкость растет. При достижении режима SCL, когда начинает доминировать дрейф носителей, емкость проходит через пик и падает. Объяснение наблюдаемых изменений $C-V$-характеристики при понижении температуры ее измерений может быть связано с прыжковым транспортом, когда электроны перемещаются по всем близко расположенным цепочкам наночастиц $\mathrm{Au}$, захватываются на них, распространяясь по всем направлениям $\mathrm{Si}: \mathrm{Au}$-нанослоя. Распределение наночастиц Аu по поверхности слоя неоднородно, однако большинство частиц группируются в ансамбли, имеющие форму цепочек. Размеры зазора между ансамблями в структуре меньше 2 нм, в таком случае, как утверждается в статье [5], туннелирование электронов между изолированными ансамблями с участие промежуточных частиц становится допустимым. Эффект от прыжкового транспорта, по-видимому, наблюдается в наших температурных измерениях $C-V$-характеристик, когда в соответствии с теорией перколяции при плавном изменении одного из параметров системы свойства системы меняются скачком. Порог перколяции разделяет две фазы: в одной фазе существуют конечные кластеры, в 
другой существует один бесконечный кластер. Проявление конечного кластера мы наблюдаем при температуре измерения $C-V$-характеристики $300 \mathrm{~K}$, когда площадь $p-n$-перехода $\approx 0.196$ мм²$^{2}$. Порог перколяции, при котором происходит формирование бесконечного кластера, наблюдается при температурах, меньших $280 \mathrm{~K}$. Проявлением эффекта от прыжкового транспорта может являться резкое изменение профиля $C-V$-характеристики при температурах начиная с $180 \mathrm{~K}$ (рис. $1, a, c$ ). Она становится подобной характеристике полупроводникового диода с $p-n$-переходом, где емкость достигает величины, равной $\approx 263$ пФ, в отличие от емкости, измеренной при температуре $300 \mathrm{~K}$, которая была равна $\approx 33 \Pi \Phi$, т.е. емкость увеличивалась в $\sim 8$ раз. При этом существенно изменялся и сам профиль $C-V$-характеристики. При температурах ниже $180 \mathrm{~K}$ с ростом напряжения обратного смещения практически наблюдалась только ее диффузионная составляющая. Слой, состоящий из $\mathrm{Si}: \mathrm{Au}-$ наночастиц образовывал бесконечный кластер, проявляющий металлические свойства в латеральной плоскости, параллельной плоскости $n$ - $\mathrm{Si}: \mathrm{Au}-p$-Si-гетерограницы, что приводило к увеличению площади $p-n$-перехода, которая достигала площади всего образца $\approx 1.68 \mathrm{mM}^{2}$. Отношение площадей $p-n$-перехода для двух температур 300 и $180 \mathrm{~K}$ в таком случае оказывается равным 8.6, что соответствует росту емкости при низких температурах измерения $C-V$-характеристики. В работе [12] при исследовании плоских конденсаторных структур на основе гранулированных пленок, состоящих из нанометрических ферромагнитных зерен, встроенных в изолирующую матрицу $\mathrm{Al}_{2} \mathrm{O}_{3}$, наблюдали явление резистивного переключения, которое сопровождалось дискретным переключением емкости. В [13] была разработана модель для туннельной проводимости в гранулированном слое, включающая процесс переноса заряда с заряженной гранулы на ближайшую нейтральную гранулу. Эффективные кинетические уравнения для усредненных плотностей заряда были выведены для характерных областей зернистого образца: областей контакта под металлическими токоподводами и свободным пространством между этими выводами. Показано, что туннельная проводимость в свободной области не вызывает заметного накопления заряда, и режим проводимости будет чисто омическим. В то же время проводимость в области контакта будет невозможной без аккумуляции там заряда, что должно привести к неомическому режиму проводимости, так как площадь контакта доминирует в общем сопротивлении. Скорость туннелирования через гранулированный слой может быть на несколько порядков медленнее, чем вдоль него, т.е. практически нечувствительна к температуре, в отличие от туннелирования через гранулированный слой, где она была сильной. Эти особенности туннелирования объяснялись различными корреляциями между соседними гранулами в одном и том же слое и в смежных слоях [14]. Для $C-V$-характеристик гетерострутур с $p-n$-переходом, в слоях которых содержатся локализованные состояния

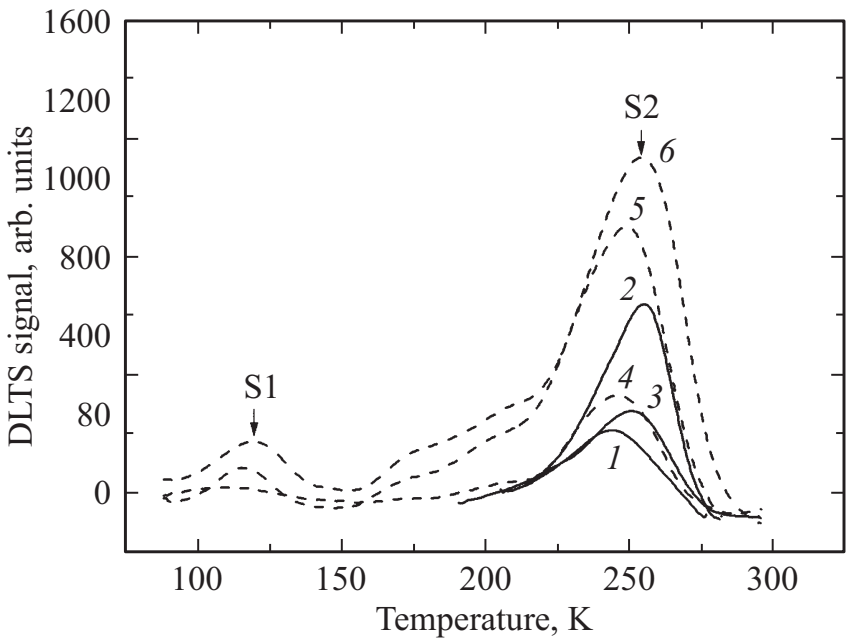

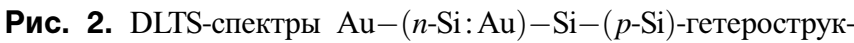
туры, полученной лазерным электродиспергированием с темпом окон $200^{-1}$ с, при напряжении импульса заполнения $V_{f}=0.10$ В и напряжениях обратного смещения $U_{r}, \mathrm{~B}: 1-$ $1.25,2-0.49,3-0.19-$ в темноте; $4-0.19-$ при освещении белым светом; при $U_{r}=0.19 \mathrm{~B}$ и $U_{f}$, В: $5-0.65$, $6-1.02-$ при освещении белым светом.

в области базы и интерфейса, обычно наблюдается гистерезис при росте напряжения смешения в сторону его роста или уменьшения и рост величины емкости при ее измерении со светом и без света [15]. На рис. 1, $b, c$ показаны подобные зависимости для исследуемых нами гетероструктур $\mathrm{Au}-($ nano- $\mathrm{Si}-\mathrm{Au})-\mathrm{Si}-(p-\mathrm{Si})$.

Для гетероструктуры $\mathrm{Au}-($ nano- $\mathrm{Si}-\mathrm{Au})-\mathrm{Si}-(p-\mathrm{Si})$ были проведены DLTS-измерения. Спектры DLTS, показанные на рис. 2, были получены при различных значениях напряжений импульса $U_{b}$, при которых производится измерение DLTS-спектров, и импульса заполнения $U_{f}$. При варьировании величины $U_{b}$ в диапазоне обратных смещений от 0.20 до $-1.58 \mathrm{~B}$ в спектрах DLTS в темноте наблюдался S1 пик, амплитуда которого достигала максимума при $U_{b}=0.49 \mathrm{~B}$ и падала при $U_{b}>-0.49 \mathrm{~B}$ и $U_{b}<-0.49 \mathrm{~B}$. При $U_{b}$ в диапазоне $(-0.20-0.20)$ В появлялся пик S2. Амплитуды обоих пиков S1 и S2 увеличивались при подсветке белым светом, которая включалась при измерении DLTS-спектров (рис. 2). Все это означает, что пики S1 и S2, проявляющиеся в DLTS-спектрах при включении импульса смещения и оптической подсветки, связаны с эмиссией электронов из ловушек, локализованных на гетерогранице nano-Si-( $p$-Si). Параметры глубоких ловушек $\mathrm{S} 1$ и S2 (энергии термической активации носителей $\left(E_{a}\right)$ и сечения их захвата на ловушки $\left.\left(\sigma_{e}\right)\right)$, определенные с помощью зависимости Аррениуса, были равны соответственно: $E_{a}=281 \mathrm{MэB}, \sigma_{e}=3.79 \cdot 10^{-18} \mathrm{~cm}^{2}$ и $E_{a}=214 \mathrm{мэB}, \sigma_{e}=1.33 \cdot 10^{-14} \mathrm{~cm}^{2}$. Измерения DLTSспектров проводились при $U_{f}=1.26 \mathrm{~B}$ и $U_{b}=0.10 \mathrm{~B}$. Обнаруженные дефекты с ГУ локализованы в области гетерограницы nano-Si-( $p$-Si) и, по-видимому, они ответственны за изменения $C-V$-характеристик таких, 
как появление пика при $U_{b}=0.05 \mathrm{~B}$, гистерезисные явления при росте напряжения смешения в сторону его роста или уменьшения, и рост величины емкости при измерении со светом и без света [15].

\section{4. Заключение}

Таким образом, результаты температурных исследований $C-V$-характеристик гетероструктуры $\mathrm{Au}-(n-\mathrm{Si}: \mathrm{Au})-\mathrm{Si}-(p-\mathrm{Si})$, на основе композитного слоя $n$-Si:Au с соотношением объемных долей наночастиц кремния и золота 33\%:67\% позволили обнаружить, что при комнатной температуре $(300 \mathrm{~K})$ эта структура проявляет свойства транзистора, включенного по схеме с общим эмиттером и отключенной базой, где базой явля-

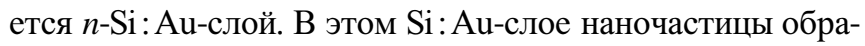
зуют конечные кластеры, где туннельная проводимость в свободной области не вызывает заметного накопления заряда, и режим проводимости будет прыжковым. В то же время в области контакта наблюдается аккумуляция заряда (рис. 1), что приводит к неомическому режиму проводимости. Эмиттерный переход транзистора образован барьером Шоттки между контактом золота и $n$-( $\mathrm{Si}: \mathrm{Au})$-слоем. Коллекторный $p-n$-переход состоит из базового $n$-nano- $(\mathrm{Si}-\mathrm{Au})$-слоя и $\mathrm{Si}$-подложки $p$-типа. При понижении температуры ниже $180 \mathrm{~K}$ происходят кардинальные изменения физических свойств слоя наночастиц $(\mathrm{Si}: \mathrm{Au})$, когда в результате эффекта перколяции система из фазы конечного кластера переходит в фазу бесконечного кластера, проявляющего металлические свойства в латеральной плоскости, параллельной

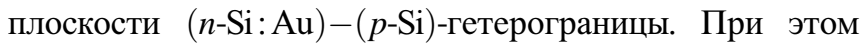
толщина базового слоя резко уменьшается, исчезает барьер Шоттки, и соответственно структура становилась $p-n$-диодом с тонкой базой, образованной подслоем, состоящим из наночастиц аморфного кремния.

Исследуемые нами $\mathrm{Au}-(n-\mathrm{Si}: \mathrm{Au})-\mathrm{Si}-(p-\mathrm{Si})$-гетероструктуры могут быть применены как для фотодетекторов с внутренним усилением сигнала, демонстрирующих большие значения спектральной чувствительности, так и для детектирования слабых оптических сигналов. Полученные результаты температурных $C-V$-характеристик $\mathrm{Au}-(n-\mathrm{Si}: \mathrm{Au})-\mathrm{Si}-(p-\mathrm{Si})$ гетероструктуры позволили обнаружить, что структура при $300 \mathrm{~K}$ может быть применена в качестве фототразистора, при освещении базы которого будет происходить генерация неравновесных электронов и дырок. Дырки будут уходить из базы в коллектор, которая будет заряжаться отрицательно относительно эмиттера, что эквивалентно прямому смещению эмиттерного перехода фототранзистора. Появляется ток инжекции из эмиттера в базу. В таком случае при коэффициенте передачи эмиттерного тока $\alpha$ в базе рекомбинирует $(1-\alpha)$ инжектированных носителей и ток транзистора будет равен $I=\left(I_{f}+I_{\mathrm{cbo}}\right) /(1-\alpha)$, где $I_{f}-$ фототок коллекторного перехода, $I_{\mathrm{cbo}}$ - обратный ток коллектора, т. е. должно происходить усиление фототока в $1 /(1-\alpha)$ раза [9].

\section{Конфликт интересов}

Авторы заявляют, что у них нет конфликта интересов.

\section{Список литературы}

[1] R.H. Chen, K.K. Likharev. Appl. Phys. Lett., 72, 61 (1998).

[2] O.S. Ken, D.A. Yavsin, P.A. Dementev, S.A. Gurevich, O.M. Sreseli. Phys. Status Solidi A, 213 (11), 2906 (2016).

[3] V.M. Kozhevin, D.A. Yavsin, V.M. Kouznetsov, V.M. Busov, V.M. Mikushkin, S.Yu. Nikonov, S.A. Gurevich, A. Kolobov. J. Vac. Sci. Technol. B, 18, 1402 (2000).

[4] М.П. Тепляков, О.С. Кен, Д.Н. Горячев, О.М. Сресели. ФТП, 52, 1071 (2018).

[5] В.И. Козуб, В.М. Кожевин, Д.А. Явсин, С.А. Гуревич. Письма ЖЭТФ, 81, 287 (2005).

[6] М.М. Соболев, О.С. Кен, О.М. Сресели, Д.А. Явсин, С.А. Гуревич. Письма ЖТФ, 44 (8), 47 (2018).

[7] М.М. Соболев, А.Р. Ковш, В.М. Устинов, А.Ю. Егоров, А.Е. Жуков, Ю.Г. Мусихин. ФТП, 33 (2), 184 (1999).

[8] N. Sengouga, R. Boumaraf, R.H. Mari, A. Meftah, D. Jameel, N. Al Saqri, M. Azziz, D. Taylor, M. Henini. Mater. Sci. Semicond. Processing, 36, 156 (2015).

[9] С. Зи. Физика полупроводниковых приборов (М., Мир, 1984) кн. 1, с. 455.

[10] A. Sharma, P. Kumar, B. Singh, S.R. Chaudhuri, S. Ghosh. Appl. Phys. Lett., 99, 023301 (2011).

[11] Durgesh C. Tripathi, Y.N. Mohapatra. Appl. Phys. Lett., 102, 253303 (2013).

[12] H. Silva, H.L. Gomes, Yu.G. Pogorelov, P. Stallinga, D.M. de Leeuw, J.P. Araujo, J.B. Sousa, S.C.J. Meskers, G. Kakazei, S. Cardoso, P.P. Freitas. Appl. Phys. Lett., 94, 202107 (2009).

[13] Y.G. Pogorelov, H.G. Silva, J.F. Polido. Phys. Rev. B, 83, 115429 (2011).

[14] G.N. Kakazei, P.P. Freitas, S. Cardoso, A.M.L. Lopes, M.M. Pereirade Azevedo, Yu.G. Pogorelov, J.B. Soma. IEEE Trans. Magn., 35, (5), 2895 (1999).

[15] T.K. Woodward, T.E. Schlesinger, T.C. McGill. Appl. Phys. Lett., 47, 631 (1985).

Редактор Г.А. Оганесян 
Impact of percolation effect on

temperature dependences of the

capacitance-voltage characteristics of

heterostructures based on composite

layers of silicon and gold nanoparticles

M.M. Sobolev, D.A. Yavsin, S.A. Gurevich

loffe Institute,

194021 St. Petersburg, Russia

Abstract The temperature dependences of the capacitancevoltage characteristics and the deep-level spectra of the $\mathrm{Au}-n-\mathrm{Si}$ : $\mathrm{Au}-\mathrm{Si}-p$-Si heterostructure based on the composite layer of $\mathrm{Au}$ and $\mathrm{Si}$ nanoparticles are studied. At a temperature of $300 \mathrm{~K}$, the structure exhibits the properties of a transistor connected in a circuit with a common emitter and a disconnected base and with a Schottky emitter barrier between the point contact $\mathrm{Au}$ and the $n$ - $\mathrm{Si}: \mathrm{Au})$ layer. In this layer, nanoparticles form finite clusters, where the conductivity will be hopping, and charge accumulation is observed in the region of the Au contact. At a measurement temperature below $180 \mathrm{~K}$, as a result of the percolation effect, the system moves from the phase of the final cluster to the phase of an infinite cluster exhibiting metallic properties in the lateral plane of the heterostructure, which turns into a $p-n$-diode. 